\title{
Necrotizing Enterocolitis with Perforation in an Extreme Preterm, Extremely Low Birth Weight Neonate
}

\author{
Gopalakrishnan $\mathbf{S}^{1}$, Singh $\mathrm{S}^{2}$, Sodhi $\mathrm{K}^{3}$, Arora $\mathbf{M}^{4}$
}

${ }^{1}$ Dr. Shridhar Gopalakrishnan, MBBS, MD, DM (Neonatology), Department of Paediatrics, ${ }^{2} \mathrm{Dr}$. Shailendra Singh, MBBS, Department of Paediatrics, ${ }^{3} \mathrm{Dr}$. Kirandeep Sodhi, MBBS, $M D$, Department of Paediatrics, ${ }^{4} \mathrm{Dr}$. Manu Arora, MBBS, MS, MCh, Department of Paediatric Surgery. All from the Command Hospital (Western Command), Chandimandir, Panchkula, Haryana, India.

\section{Address for correspondence:}

Dr. Manu Arora

E-mail:drmanuarora@gmail.com

\section{How to cite}

Gopalakrishnan S, Singh S, Sodhi K, Arora M. Necrotizing Enterocolitis with Perforation in an Extreme Preterm, Extremely Low Birth Weight Neonate. J Nepal Paediatr Soc 2016;36(2):201203.

doi: http://dx.doi.org/10.3126/jnps.v36i2.15199

This work is licensed under a Creative Commons Attribution 3.0 License.

\begin{abstract}
Necrotizing enterocolitis (NEC) remains the most common and serious surgical condition among preterm, very low birth weight neonates with a mortality of $40 \%$ to $50 \%$ in those requiring surgery. We report perforation secondary to NEC in an extreme preterm, extremely low birth weight neonate with a good outcome due to aggressive surgical management and intensive care.
\end{abstract}

Key words: Necrotizing enterocolitis; Pneumoperitoneum; feeding; Surgical management

\section{Introduction}

$\mathrm{N}$ ecrotizing enterocolitis (NEC) is a potential surgical emergency with the majority of cases occurring in very preterm (<32 wks), very low birth weight $(<1500 \mathrm{~g})$ neonates ${ }^{1}$. The presentation can be insidious over a few days with abdominal distension, altered gastric aspirates, feed intolerance along with systemic symptoms or it can present as a catastrophic deterioration with perforation and multiorgan dysfunction ${ }^{2}$. The age at onset is around $11-12$ days and is inversely related to the postmenstrual age at birth ${ }^{3}$. Current research is focussed on prevention and early recognition of NEC and the most promising approach seems to be the role of probiotics ${ }^{4}$. We report a case of NEC with perforation in an extreme preterm, extremely low birth weight (ELBW) neonate on day five after birth that was successfully managed with surgical resection and had a good outcome.

\section{The Case}

An extreme preterm 27 weeks, ELBW $880 \mathrm{~g}$, female neonate was born by spontaneous vaginal delivery to a 23-year-old primigravida mother who had premature prolonged rupture of membranes ( $p$ PROM) for eight days and presented with antepartum haemorrhage three days prior to delivery. An antenatal ultrasound four weeks ago had shown severe oligohydramnios and placenta previa. She had not received any antenatal steroids (ANS) and delivered en-route from a peripheral hospital in the ambulance. The neonate was born severely asphyxiated and received bag and mask ventilation for three min and shifted to the neonatal intensive care unit. In view of early onset respiratory distress, nasal continuous positive airway pressure (CPAP) was begun and early rescue surfactant was administered. Intravenous (IV) 10\% dextrose, 10\% Aminoven and IV antibiotics 
(Cefotaxime and Amikacin) were begun in view of maternal pPROM.

The neonate remained stable and minimal enteral nutrition (MEN) with expressed breastmilk was started on day two after birth. The respiratory distress settled and CPAP was weaned off by day fourafter birth. However abdominal distension with altered/bilious nasogastric aspirateswas noted by day five after birth. Over the next $24 \mathrm{hrs}$ there were recurrent episodes of apnoea and increasing oxygen requirement and the neonate required mechanical ventilation. The neonate was kept nil per oral on continuous nasogastric aspiration and antibiotics were upgraded to Meropenem and Metronidazole. $X$ ray abdomen revealed evidence of intestinal perforation (Figure 1) and the neonate was taken up for exploratory laparotomy. Per operatively, there was a jejunal perforation and a resection anastomosis was done. The neonate required mechanical ventilation for three days and was successfully extubated to nasal CPAP and weaned to room air by day 12 after birth. The postoperative course was complicated by culture positive late onset sepsis ( $E$ Coli and coagulase negative staphylococcus(CoNS) in blood culture, no meningitis) which was treated with two weeks of IV antibiotics and feed intolerance requiring prolonged parenteral nutrition and gradual increase in feed volume along with antireflux medications. At discharge, the infant weighed $1600 \mathrm{~g}$ and was on expressed breast milk along with human milk fortifier/vitamin D3/ Iron supplements and had normal retinopathy of prematurity (ROP), ultrasound skull and hearing screens.

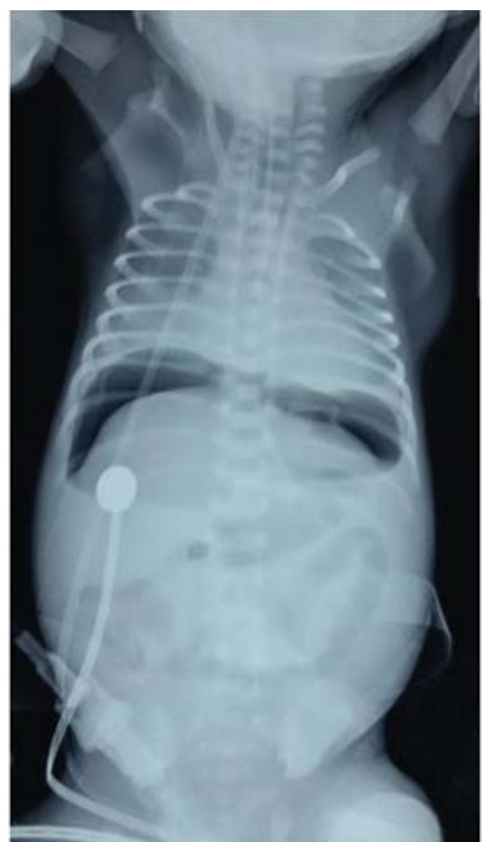

Fig 1: X-ray abdomen erect view: Air under diaphragm suggestive of intestinal perforation

\section{Discussion}

Necrotizing enterocolitis, despite advances in neonatal care is still associated with a high incidence of morbidity and mortality around $20-40 \% 5$. The indications for surgery include evidence of perforation, as was seen in our index case and presence of necrotic bowel as evidenced by severe and persistent metabolic acidosis and/or thrombocytopenia and a lack of improvement with medical management ${ }^{5}$.

In our index case, the mother had not received ANS and the neonate had severe perinatal asphyxia at birth. There is still controversy with regard to initiation of feeds in such extremely preterm, ELBW neonates with the current evidence favouring MEN with human milk at the earliest sign of clinical stability ${ }^{6,7}$. We had initiated expressed breast milk at $10 \mathrm{ml} / \mathrm{kg}$ after 24 hours as the neonate had passed meconium and was clinically and haemodynamically stable. The feeds were increased to $20 \mathrm{ml} / \mathrm{kg}$ after two days and the neonate became symptomatic by day five after birth. The neonate was managed aggressively with IV Fluids/parenteral nutrition, IV antibiotics and mechanical ventilation. As the $\mathrm{x}$ ray showed evidence of air under the diaphragm, an exploratory laparotomy was done immediately after stabilization. The neonate was successfully operated with resection of jejunal segment and primary reanastomosis after confirming the site of perforation in the jejunum. The post-operative period was marked by late onset sepsis, feed intolerance and the neonate required prolonged parenteral nutrition. There was however no neonatal cholestasis. The overall mortality for ELBW neonates that undergo surgery for NEC has been reported to be $40 \%$ to $50 \%$ despite intensive care $^{8,9,10}$.

\section{Conclusion}

This case highlights the need for close clinical monitoring for signs of NEC and early recognition of the need for surgery in such an extreme preterm, ELBW neonate. The prompt and successful surgical management and aggressive neonatal intensive care resulted in a favourable outcome. Early recognition and prompt surgical management of perforation in NEC along with neonatal intensive care is crucial for a good outcome. 


\section{References}

1. Thompson AM, Bizzarro MJ. Necrotizing enterocolitis in newborns: pathogenesis, prevention and management. Drugs 2008;68(9):1227-38.

2. Neu J, Walker WA. Necrotizing enterocolitis. N Engl J Med 2011;20;364(3):255-64.

3. Sharma R, Hudak ML, Tepas JJ, Wludyka PS, Marvin WJ, Bradshaw JA, et al. Impact of gestational age on the clinical presentation and surgical outcome of necrotizing enterocolitis. J Perinatol Off J Calif Perinat Assoc 2006;26(6):342-7.

4. AlFaleh K, Anabrees J. Probiotics for prevention of necrotizing enterocolitis in preterm infants. Cochrane Database Syst Rev 2014;4:CD005496.

5. Sharma R, Hudak ML. A clinical perspective of necrotizing enterocolitis: past, present, and future. ClinPerinato/2013;40(1):27-51.

6. Morgan J, Bombell S, McGuire W. Early trophic feeding versus enteral fasting for very preterm or very low birth weight infants. Cochrane Database Syst Rev 2013;3:CD000504.

7. Quigley M, McGuire W. Formula versus donor breast milk for feeding preterm or low birth weight infants. Cochrane Database Syst Rev 2014;4:CD002971.

8. Blakely ML, Lally KP, McDonald S, Brown RL, Barnhart DC, Ricketts RR, et al. Postoperative outcomes of extremely low birth-weight infants with necrotizing enterocolitis or isolated intestinal perforation: a prospective cohort study by the NICHD Neonatal Research Network. Ann Surg 2005;241(6):984-9; discussion 989-94.

9. Henry MCW, Moss RL. Neonatal necrotizing enterocolitis. SeminPediatrSurg2008;17(2):98-109.

10. Shah SSH, Saleem M, Mehmood T, Ahmed T. Frequency and outcome of necrotizing enterocolitis in preterm neonates. J Ayub Med Coll Abbottabad JAMC. 2015;27(1):85-7. 